Relato de Caso

\title{
Tratamento Restaurador de Lesões Cervicais Cariosas Associado a Ações Preventivas: Relato de Caso
}

Restoring Treatment of Cariosis Cervical Injuries Associated With Preventive Actions: Case Report

Alice Castro Guedes Mendonça1; Basílio Rodrigues Vieira²; José Henrique de Araújo Cruz; Eugênia Lívia de Andrade Dantas 3 ; Moan Jéfter Fernandes Costa ${ }^{4}$; Gymenna Maria Tenório Guênes ${ }^{5}$

${ }^{1}$ Graduanda, curso de Odontologia, Universidade Federal da Paraíba, João Pessoa-PB.

2Mestrando, Programa de Pós-graduação em Odontologia, Universidade Federal da Paraíba, João Pessoa-PB.

${ }^{3}$ Graduando, curso de Odontologia, Universidade Federal de Campina Grande, Patos-PB.

${ }^{4}$ Mestrando, Programa de Pós-graduação em Saúde Coletiva, Universidade Federal do Rio Grande do Norte, Natal-RN.

${ }^{5}$ Professora do curso de Odontologia, Universidade Federal de Campina Grande, Patos-PB.

Autor para correspondência:

Basilio Rodrigues Vieira. Rua Anizio de Azevedo Lima, oㅡ 212, Mangabeira II, João Pessoa,

Paraíba. CEP: 58057-270. E-mail: Basilio_451@hotmail.com

\begin{abstract}
Resumo
Introdução: A ocorrência de lesões cariosas na região cervical é comumente encontrada, particularmente pela capacidade de acúmulo de biofilme nesta área. Objetivo: Descrever o tratamento restaurador de lesões cariosas classe $\mathrm{V}$ de Black em elementos dentais anteriores e posteriores, associado a ações de promoção em saúde bucal. Relato do caso: Paciente, gênero feminino, comparaceu a Unidade Básica de Saúde relatando incômodo estético nos elementos dentais após remoção de aparelho ortodôntico. Ao exame clínico foram observadas lesões cariosas nas cervicais dos elementos $13,23,24,33,34,35$ e 43 , além de mancha branca ativa nos elementos superiores anteriores e inferiores. Foi realizado evidenciação de placa, orientação de higiene bucal $(\mathrm{OHB})$ e aplicação de $\mathrm{NaF}$ a $2 \%$ neutro seguido de três sessões clínicas de tratamento restaurador com resina composta pela técnica convencional, com posterior acabamento e polimento das restaurações. Na última sessão realizou-se nova evidênciação de placa, $\mathrm{OHB}$ e aplicação de $\mathrm{NaF}$ a $2 \%$. Conclusão: Um tratamento restaurador de lesões cariosas associadas a ações de promoção de saúde motivaram a paciente ao cuidado da saúde bucal, reduzindo as chances das lesões de cárie se estenderem em direção à polpa, diminuindo o risco de cáries secundárias e culminando com o sucesso estético, biológico e funcional.
\end{abstract}

Descritores: Dentística. Restauração dentária permanente. Resinas compostas. 


\begin{abstract}
Introduction: The occurrence of carious lesions in the cervical region is commonly found, particularly due to the capacity of biofilm accumulation in this area. Objective: To describe restorative treatment in conjunction with Black class $\mathrm{V}$ carious lesion promoter in anterior dental elements and later. Case report: Patient, female, compared the Basic Health Unit reporting aesthetic discomfort in the dental elements after removal of orthodontic appliance. Clinical examination revealed carious cervical lesions of elements $13,23,24,33,34,35$ and 43 , as well as an active white spot on the anterior and inferior upper elements. It was performed plaque disclosure, oral hygiene orientation $(\mathrm{OHB})$ and application of neutral $2 \% \mathrm{NaF}$ followed by three clinical sessions of restorative treatment with resin composed by the conventional technique, with subsequent finishing and polishing of the restorations. In the last session, new evidence of plaque, HBs and application of $2 \% \mathrm{NaF}$ was performed. Conclusion: A restorative treatment of carious lesions associated with health promotion actions motivated the patient to care for oral health, reducing the chances of caries lesions extending towards the pulp, reducing the risk of secondary caries and culminating with aesthetic success, biological and functional.
\end{abstract}

Key-words: Dentistry. Permanent dental restoration. Composite resins.

\title{
Introdução
}

A cárie dentária é uma das doenças mais comuns do mundo, possui aspecto multifatorial abrangendo fatores primários (biofilme), determinantes (açúcares e fluoretos) e moduladores (fatores biológicos e sociais) ${ }^{1}$. O Brasil é considerado um país com altos índices de prevalência em doenças orais, principalmente a cárie. Um dos influenciadores da cárie é o fator socioeconômico, em virtude da baixa renda que está atrelado ao grau de educação, estilo de vida e o acesso à informação².

A partir disso, os cirurgiões-dentistas possuem o dever de influenciar a qualidade de vida dos pacientes, assegurando por todos os meios existentes e disponíveis uma cavidade bucal saudável, atingindo este objetivo através da prevenção das doenças bucais ${ }^{3}$.

Para fortalecer a saúde bucal, uma das alternativas são os tratamentos fluorterápicos, pois manter a substantividade do flúor proporciona ao paciente remineralização dos dentes atingidos pelo processo carioso ${ }^{4}$, permitindo a aplicação e uso em todas as fases do tratamento, tanto pelo cirurgião-dentista como pelo próprio paciente ${ }^{5}$.

A perda de tecido duro atingida por cárie na região cervical dos dentes é muito comum e tem sua prevalência e severidade aumentada com a idade ${ }^{6}$. Tal fato ocorre devido a menor espessura de esmalte/cemento na região cervical fazendo com que a superfície dentinária seja exposta ao meio oral?. 
As restaurações diretas em resina composta para os dentes anteriores e posteriores tem crescido em importância devido aos avanços nas formulações destes materiais e sistemas adesivos que tornam a aplicação de restaurações nestes dentes apropriadas sob o ponto de vista estético, biológico e funcional ${ }^{8-10}$.

O objetivo deste trabalho é descrever o tratamento integrado (restaurador e de promoção à saúde) de lesões cariosas na região cervical de elementos dentais anteriores e posteriores com o uso de resina composta e paralisação de lesões cariosas ativas (nível subclínico) por meio da fluorterapia.

\section{Relato de Caso}

Paciente de 22 anos de idade, gênero feminino, comparaceu à Unidade Básica de Saúde para atendimento odontológico queixando-se de incômodo estético nos elementos dentais após remoção de aparelho ortodôntico. Ao exame clínico intraoral, foi constatado que se tratava de lesões cariosas nas cervicais dos elementos 13, 23, 24, 33, 34, 35 e 43, além de mancha branca ativa nos elementos superiores anteriores e inferiores.

O planejamento, em acordo com o paciente, baseou-se na execução de evidenciação de placa na primeira sessão, com finalidade de orientar a paciente sobre as áreas com maior acúmulo de biofilme e emprego da escovação mais adequada visando motiva-la para o cuidado e manutenção da sua saúde bucal (Figura 1). Foi realizada a aplicação tópica de Fluorfostato Acidulado 1,23\% (Nova DFL, Brasil) para inativação e remineralização das lesões cariogênicas ativas.

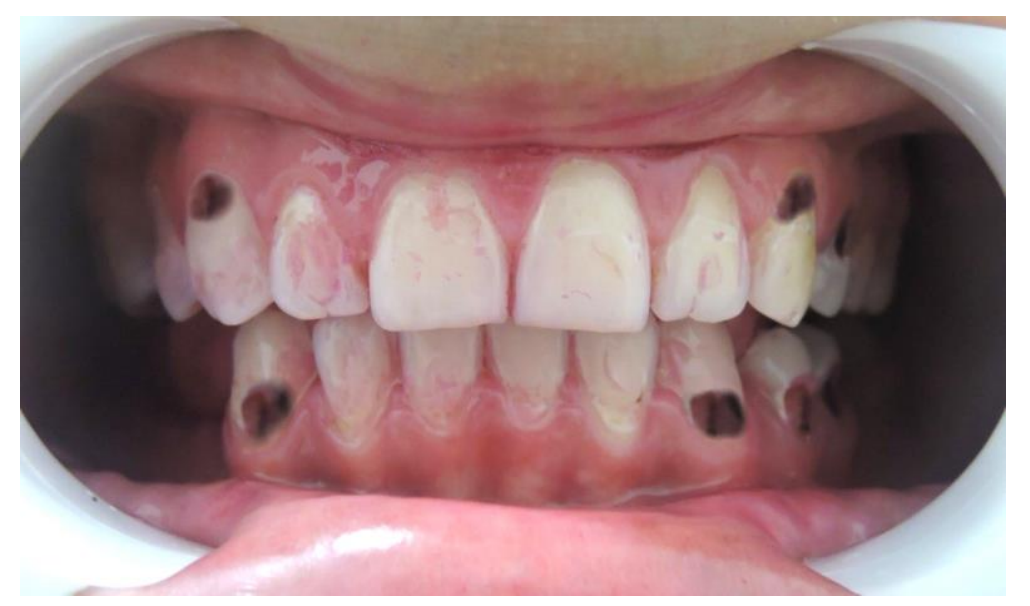

Figura 1. Aspecto inicial após evidenciação de biofilme. 
O tratamento restaurador foi realizado em três sessões clínicas com remoção de tecido dentário cariado sob alta rotação e boa refrigeração com pontas diamantadas esféricas (KG, Brasil), além da remoção de dentina afetada com brocas esféricas em baixa rotação (CA Maillefer, Brasil) (Figura 2).

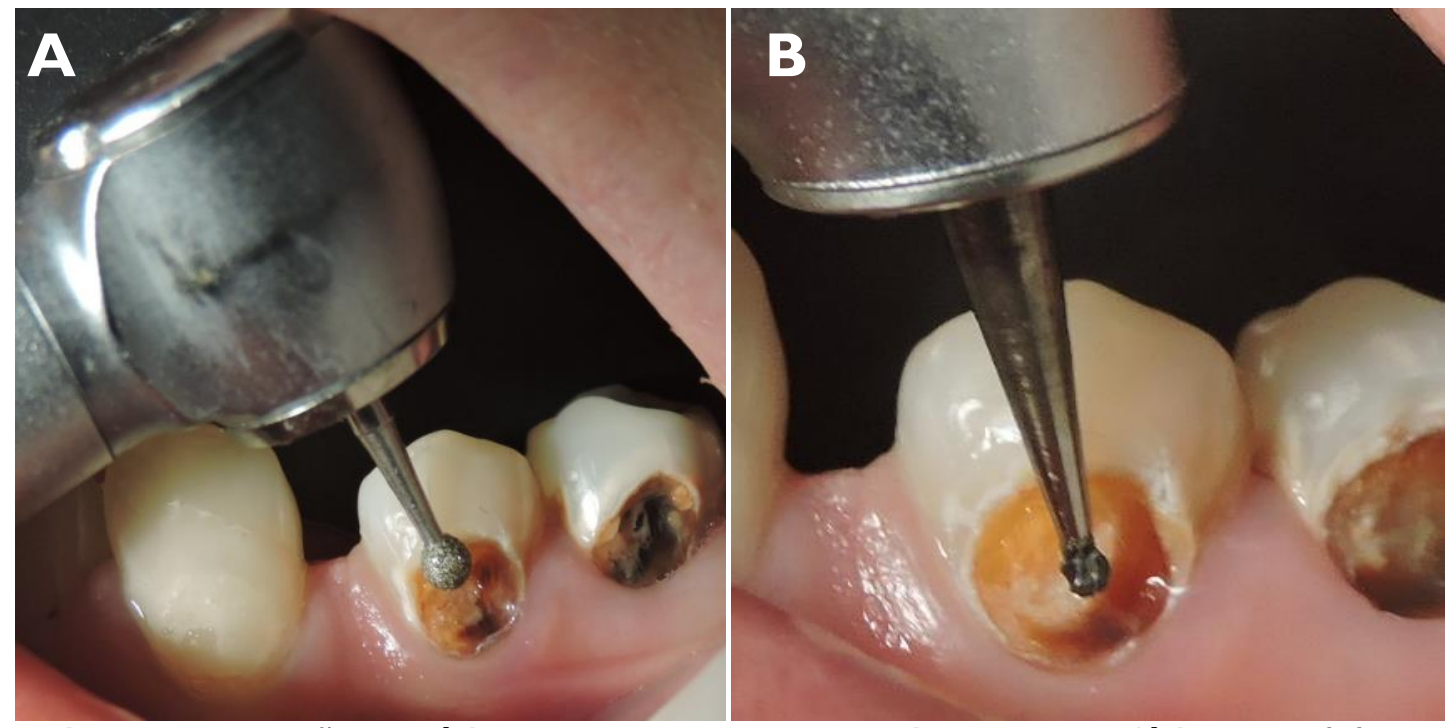

Figura 2: Remoção de cárie em esmalte com ponta diamantada esférica 1014 (A) e remoção de cárie em dentina com broca em aço esférica no 3 (B).

Subsequentemente, optou-se por utilizar um material restaurador definitivo em resina composta microparticulada na cor A3,5 (Opallis - FGM, Brasil), associada à hibridização pela técnica convencional com adesivo monocomponente (Ambar -FGM, Brasil) (Figura 3).

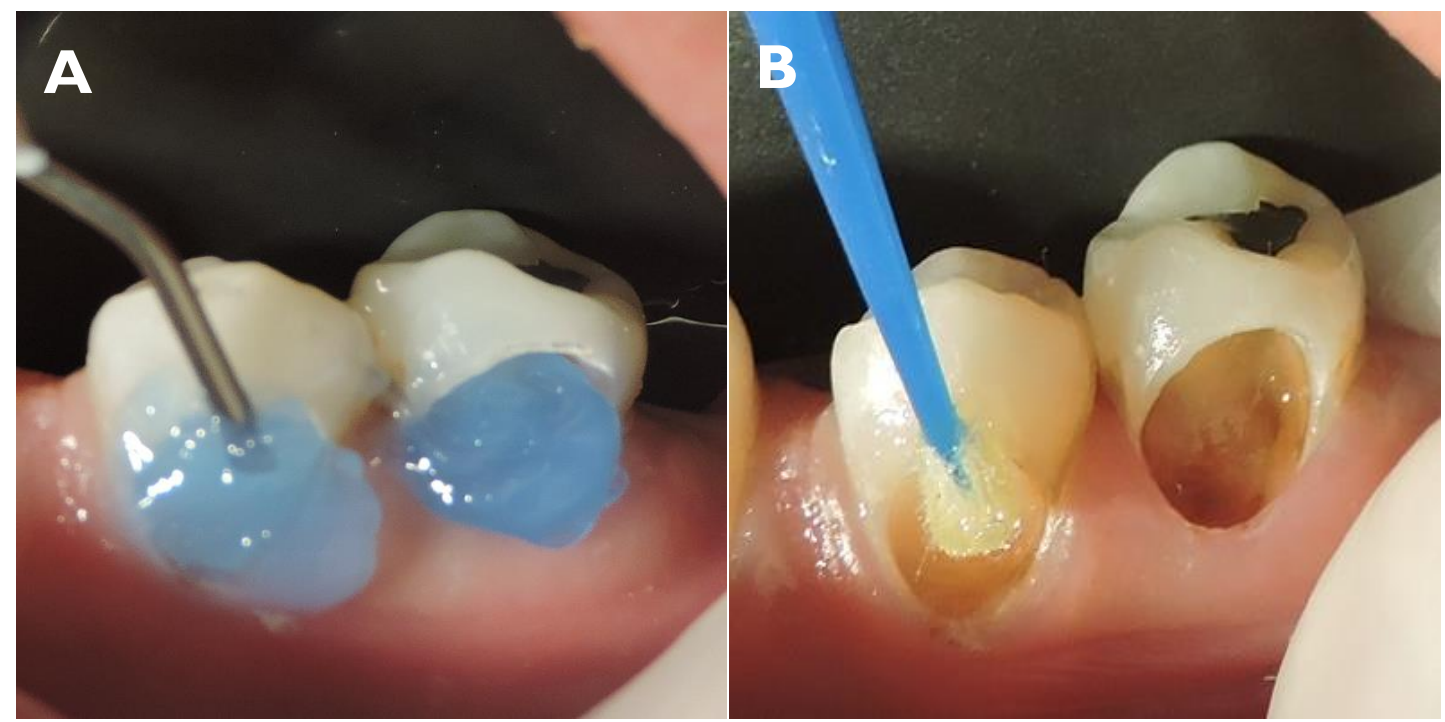

Figura 3: Aplicação de ácido fosfórico a 37\% (A) e aplicação de sistema adesivo com auxílio de aplicador tipo microbrush (B). 
Os elementos 13 e 24 que apresentaram proximidade com a cavidade pulpar receberam como material forrador Cimento de Hidróxico de Cálcio (HidroC-Dentsply, Brasil) e Cimento de lonômero de Vidro (CIV) (Maxxion-FGM, Brasil) como base, previamente ao ataque com ácido fosfórico a 37\% (Biodinâmica, Brasil). A inserção da resina composta foi realizada com espátula tipo Almore (Golgran, Brasil) pela técnica incremental (Figura 4).

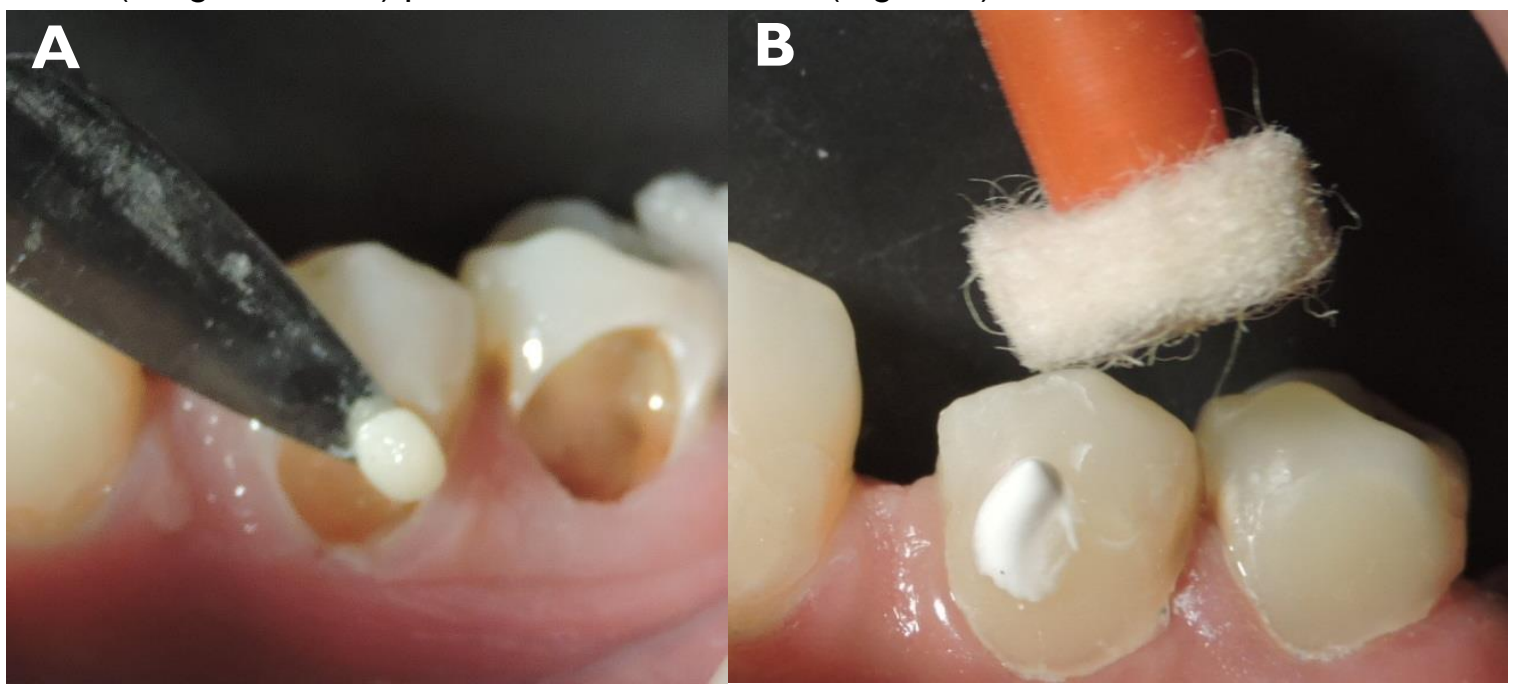

Figura 4. Inserção de resina composta pela técnica incremental (A) Procedimento de polimento das restaurações com disco de feltro e pasta polidora (B).

Nas sessões finais foram realizados procedimentos de acabamento com pontas diamantadas do tipo F e FF (KG, Brasil), tiras e disco de lixa (Microdont, Brasil) e polimento com discos de feltro (FGM, Brasil) com auxílio de pasta para polimento (Diamond,FGM, Brasil) (Figura 4), resguardandose um período de tempo de no mínimo 24h. Na última sessão foi realizada mais uma evidenciação de placa com nova orientação de higiene bucal devido a nova condição da paciente, além de nova aplicação tópica de Fluorfostato Acidulado 1,23\%, integrando reforço contra o desafio cariogênico (Figura 5).

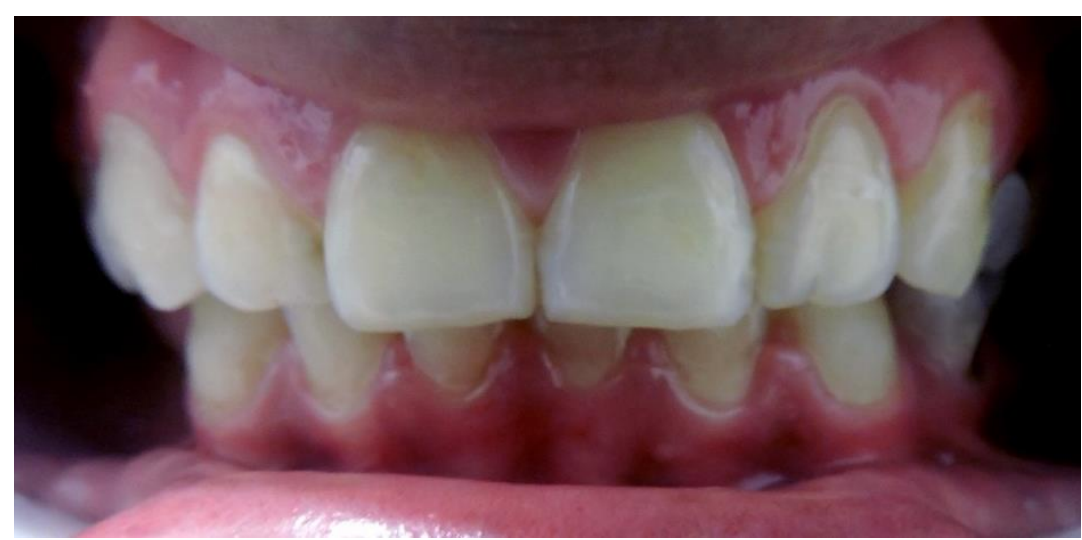

Figura 5: Aspecto final da paciente. 


\section{Discussão}

Restauração de lesões cervicais podem ser realizadas utilizando técnicas e materiais restauradores diversos, os quais são selecionados de acordo com requisitos mecânicos e/ou estéticos do caso em particular11.

Está claro que os acessórios ortodônticos colados nas superfícies dentárias complicam a higienização e atuam como precursores adicionais de biofilme, levando a desmineralizações do esmalte, causando manchas brancas, cáries dentárias e gengivite ${ }^{12}$. Mas, essa condição pode ocorrer de forma rotineira se não houver um preparo prévio dos pacientes antes da instalação do aparelho ortodôntico fixo e da falta de reforço e motivação sobre higiene oral durante o tratamento ${ }^{12}$.

As resinas compostas $(\mathrm{RC})$ são a escolha por excelência para restauração de lesões cervicais por apresentam uma grande variedade de cores e translucidez, tornando possível conseguir uma aparência estética cada vez mais natural. Além disso, a RC pode ser escolhida pelo preparo conservador que requer a conservação da estrutura dental, além da facilidade de inserção dos incrementos. Os compósitos microparticulados apresentam módulo de elasticidade menor quando comparado aos híbridos e, portanto, tendem a "flexionar" com o dente sob pressão ao invés de se desprender dele, por isso são preferidos ${ }^{11}$.

O CIV modificado por resina e os compômeros podem ser empregados em condições semelhantes, porém não são tão frequentemente utilizados quanto às resinas compostas, visto que sua inserção é mais difícil, possuem menor resistência ao desgaste, compressão e tensão mais baixas. Em contrapartida, os ionômeros possuem adesão química e mecânica à estrutura dental e liberação de flúor, que pode ser especialmente benéfico em pacientes com alto risco à cárie ${ }^{13}$.

A menor espessura do esmalte na região pode levar a uma progressão mais rápida da cárie em dentina, assim como a proximidade da cárie e presença de placa bacteriana aos tecidos gengivais pode, com a progressão da lesão, causar problemas periodontais que vão de gengivites localizadas até, em casos mais severos como perda do espaço biológico periodontal, reabsorção óssea e recessão gengival, tornando sombrio o prognóstico ${ }^{12}$.

A odontologia atual não se limita apenas ao conhecimento da prevalência da cárie dentária, mas sobretudo às resoluções dos problemas em saúde bucal nos indivíduos, procurando sempre a promoção e a prevenção de saúde ${ }^{2}$. O acesso às informações sobre saúde bucal colabora para tornar a sociedade perspicaz na manutenção de sua saúde, tendo na mudança de atitude dos pacientes o mecanismo para isso, uma vez que os hábitos adquiridos são implementados e permanecem por toda a vida ${ }^{14}$. 
A ação do fluoreto está em reduzir a velocidade com que as lesões de cárie progridem em um determinado período de tempo ${ }^{15,16}$. Então, o seu uso tópico profissional tem extrema utilidade em situações que a prevalência de cárie é muito alta ou quando o paciente apresenta um risco muito elevado de cárie, além disso, pode-se concluir que a presença de fluoretos nos dentifrícios e a utilização em larga escala pela população, tem papel na manutenção das lesões em estágio subclínico ${ }^{17-19}$. Desta forma, a fluorterapia deve ser posta como coadjuvante em qualquer programa de prevenção de cárie individualizado ${ }^{20}$.

\section{Conclusão}

Um tratamento restaurador de lesões cariosas associadas a ações de promoção de saúde motivaram a paciente ao cuidado da saúde bucal, reduzindo assim o risco de cáries secundárias, que são tidas como o maior motivo de falhas e substituição de restaurações.

\section{Referências}

1. Ferreira-Nóbilo NP, Sousa MLR; Cury, JA. Conceptualization of Dental Caries by Undergraduate Dental Students from the First to the Last Year. Braz. Dent. J. 2014;25(1):52-59.

2. Dias AP, Marques RB. Prevalência de cárie dentária em primeiros molares permanentes de crianças de 6 a 12 anos de idade. R. Interd. 2017;10(3):78-90.

3. Gigliotti MP, Theodoro D, Oliveira TM, Silva SMB, Machado MAM. Relação entre nível de escolaridade de mães e percepção sobre saúde bucal de bebês. Salusvita. 2007;26(2):65-73.

4. Silva PDC, Giffoni TCR, Matsuura E, Franzin LCS, Progiante PS, Goya S. Cárie precoce da infância, qualidade de vida e tratamento: revisão de literatura. Revista Uningá. 2015;24(3):86-89.

5. Oliveira AL, Botta A, Rosell F. Promoção de saúde bucal em bebês. Revista de Odontologia da Universidade Cidade de São Paulo. 2010;22(3):247-253

6. Simões MP, Albino LGB, Reis AF, Rodrigues JA. Restaurações estéticas con-servadoras em dentes anteriores. R Dental Press Estét. 2009;6(1):90-101.

7. Vellasco K, Campos I, Zouain-ferreira TRF, Basting RT. Dentística minimamente invasiva: plástica dental. Arq Odontol. 2006;42(2):104-112.

8. Blitz N. Direct bonding in diastema closure - drama, immediate resolution. Oral Health.1996;86(7):23-26.

9. Fahl N. Achieving ultimate anterior esthetics with a new microhybrid composite. Compendium.2000; 21(26 Suppl):4-13. 
10. Baratieri LN. Restaurações adesivas diretas com resinas compostas em dentes anteriores. Ed. Santos. São Paulo; 2002. 131p.

11. Barbosa LPB, Prado Junior RR, Mendes RF. Lesões cervicais não-cariosas: etiologia e opções de tratamento restaurador. Revista Dentística. 2009;8(18):5-10.

12. Olympio KPK, Bardal PAP, Henriques JFC. Bastos JRM. Prevenção de cárie dentária e doença periodontal em Ortodontia: uma necessidade imprescindível. Maringá. 2006;11(2):110119.

13. Haveman C, Burgess J, Summitt JB. A clinical comparasion of restorative meterials for caries in xerostomic patients. J Dent Res. 1999;78(4):628-637.

14. Abreu MHNG, Pordeus IA, Modena CM. Representações sociais de saúde bucal entre mães no meio rural de Itaúna (MG). Ciênc Saúde Coletiva. 2005;10(1):245-259.

15. Koch G, Fejerskov O, Thylstrup A. Flúor no tratamento da cárie dentária: implicações clínicas. In: THYLSTRUP, A; FEJERSKOV, O. Tratado de cariologia. 2. ed. São Paulo: Santos; 1995. p. 259-282.

16. McDonagh MS, Whiting PF, Wilson PM, Sutton AJ, Chestnutt I, Cooper J, Misso K, Bradley M, Treasure E, Kleijnen J. Systematic review of water fluoridation. Brit. Med. J. 2000;321(7265):855859.

17. Peres MAA, Rosa AGF. As causas da queda da cárie. RGO. 1995;43(3):160-164.

18. Mellberg JR. Fluoride dentifrices: current status and prospects. Int. Dent. J. 1991;41(1):9-16.

19. Kalsbeek H, Kwant GW, Groeneveld A, Backer Dirks O, van Eck AAMJ, Theuns HM. Caries experience of 15-year old children in the Netherlands after discontinuation of water fluoridation. Caries Res. 1993;27(3):201-205.

20. Almeida MBHA, Neves AA. Fluorterapia na prevenção da cárie dental. Passo Fundo. 2002;7(2):7-11. 